Article

\title{
MagnetoPlasmonic Waves/HOMO-LUMO Free $\pi$-Electron Transitions Coupling in Organic Macrocycles and Their Effect in Sensing Applications
}

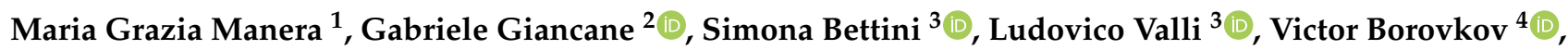 \\ Adriano Colombelli ${ }^{1}$, Daniela Lospinoso ${ }^{1}$ (D) and Roberto Rella ${ }^{1, *(D)}$ \\ 1 Istituto per la Microelettronica e Microsistemi-IMM-CNR Lecce, Via Monteroni, 73100 Lecce, Italy; \\ mariagrazia.manera@cnr.it (M.G.M.); adriano.colombelli@le.imm.cnr.it (A.C.); \\ daniela.lospinoso@le.imm.cnr.it (D.L.) \\ 2 Dipartimento di Beni Culturali, Università del Salento, Via D. Birago, 73100 Lecce, Italy; \\ gabriele.giancane@unisalento.it \\ 3 Dipartimento di Scienze e Tecnologie Biologiche e Ambientali (DiSTeBA), Università del Salento, \\ Via Monteroni, 73100 Lecce, Italy; simona.bettini@unisalento.it (S.B.); ludovico.valli@unisalento.it (L.V.) \\ 4 Department of Chemistry and Biotechnology, Tallinn University of Technology, Akadeemia tee 15, \\ 12618 Tallinn, Estonia; victor.borovkov@taltech.ee \\ * Correspondence: roberto.rella@cnr.it
}

\section{check for} updates

Citation: Manera, M.G.; Giancane, G.; Bettini, S.; Valli, L.; Borovkov, V.; Colombelli, A.; Lospinoso, D.; Rella, R. MagnetoPlasmonic Waves/ HOMO-LUMO Free $\pi$-Electron Transitions Coupling in Organic Macrocycles and Their Effect in Sensing Applications. Chemosensors 2021, 9, 272. https://doi.org/ $10.3390 /$ chemosensors 9100272

Academic Editor: Luís C. Coelho

Received: 1 September 2021

Accepted: 17 September 2021

Published: 22 September 2021

Publisher's Note: MDPI stays neutral with regard to jurisdictional claims in published maps and institutional affiliations.

Copyright: (c) 2021 by the authors. Licensee MDPI, Basel, Switzerland. This article is an open access article distributed under the terms and conditions of the Creative Commons Attribution (CC BY) license (https:/ / creativecommons.org/licenses/by/ $4.0 /)$.
Abstract: Optical and magneto-optical surface plasmon resonance (MOSPR) characterization and preliminary sensing test onto single- and multi-layers of two organic macrocycles have been performed; $\mathrm{TbPc}_{2}\left(\mathrm{OC}_{11} \mathrm{H}_{21}\right)_{8}$ phthalocyanine and $\mathrm{CoCoPo}_{2}$ porphyrin were deposited by the Langmuir-Schäfer (LS) technique onto proper $\mathrm{Au} / \mathrm{Co} / \mathrm{Au}$ magneto-optical transducers. Investigations of the MOSPR properties in Kretschmann configuration by angular modulation, gives us an indication about the potential discrimination of two organic macrocycles with absorption electronic transition in and out of the propagating plasmon energy spectral range. An improved molecular vapors sensitivity increase by the MOSPR sensing probe can be demonstrated depending on the overlap between the plasmonic probe energy and the absorption electronic transitions of the macrocycles under investigation. If the interaction between the plasmon energy and molecular HOMO-LUMO transition is preserved, a variation in the complex refractive index takes place. Under this condition, the magneto-plasmonic effect reported as 1/ I MOSPR I signal allows us to increase the detection of molecules deposited onto the plasmonic transducer and their gas sensing capacity. The detection mechanism appears strongly enhanced if the Plasmon Wave/HOMO-LUMO transitions energy are in resonance. Under coupling conditions, a different volatile organic compounds (VOC) sensing capability has been demonstrated using $n$-butylamine as the trial molecule.

Keywords: magneto-plasmonic; cobalt bisporphyrins; terbium bisphthalocyanine; Langmuir-Schäfer technique; chemical sensing

\section{Introduction}

The interaction mechanism taking place between organic macrocycles such as metallatedphthalocyanines and metallated-porphyrins and surface plasmons has been investigated to describe how plasmonic materials can interact with nearby molecules by enhancing their spectroscopic signatures, thus allowing for plasmon-enhanced sensing and detection [1-4]. Recently a lot of application based onto fluorescence technologies have been adopted that exploit single molecule sensitivity for, e.g., DNA sequencing [5] and immunoassays based on single enzymes and single-molecule counting [6], or improving and targeted delivery of bioactive molecules to cells with magnetic layer-by-layer assembled microcapsules [7], or biosensor based on magneto-resistance technology [8]; a modelling of magneto impedance response of thin film sensitive element in the presence of ferrogel as 
a step toward development of biosensor for in tissue embedded magnetic nanoparticles detection [9].

Important groups of compounds containing an aromatic macrocycle with alternating double and single bonds are porphyrins and phthalocyanines [10,11]. The first class of proposed macromolecules are ubiquitous in Nature and can be regarded as derivatives of the heterocycle porphyrin and differ in the chemical groups on the periphery and central metal atom, in our study cobalt. The latter are highly colored and the substitution of the central hydrogen atoms by metal ones, in our case terbium, results in the changes of the optical properties in the UV-VIS spectral range. By this strategy they can be deposited by chemical deposition methods like the Langmuir-Blodgett deposition technique onto a proper transducer [12-14]. The interest in these macrocycles is due to the peculiar properties of the large family of tetrapyrrolic functional molecular materials, above all their high chemical and thermal stability. These compounds generated a lot of interest for chemists, physicists, and industrial scientists due to their role in growing technologies including photoconductors [15], solar cells [16,17] and chemical sensors [18-21]. Generally, Langmuir-Blodgett (LB) technique involves the vertical movement of a solid substrate through the monolayer/air interface and ensures a thorough control over single monolayer deposition parameters. In our case well organized monolayers have been deposited by using the horizontal lifting or Langmuir-Schäfer (LS) technique [22-24] a well-established method of controlling thickness and molecular orientation within thin organic film $[25,26]$.

In this work, the two macrocycles have been deposited in monolayer and multilayer thin film form by the LS method and characterized during and after deposition by optical approaches. In particular, the interest is related to the optical behavior and energy transfer taking place between the two-metal coordinated organic macrocycles deposited in thin film form and the propagating surface plasmon magnetically activated in a Kretschmann configuration. Generally, these interactions can be investigated in wavelength or angular modulation Kretschmann configuration. In our case angular modulation configuration has been adopted. Recently, the magneto-optical surface plasmon resonance (MOSPR) technique has been proposed based on the interrelation of magneto-optic effects and surface plasmon resonance (SPR) excited in multilayers of noble (such as gold or silver) and ferromagnetic metals (such as cobalt or iron). Experimental evidence has already led to the application of this technique as a novel gas sensing method for the monitoring of refractive index changes at the metal-dielectric interface.

In this paper, we report about the strong interaction between molecular excitations in $\mathrm{CoCoPo}_{2}$ and $\mathrm{TbPc}_{2}\left(\mathrm{OC}_{11} \mathrm{H}_{21}\right)_{8}$ molecules and magnetically modulated propagating SPR excitations. We observe a strong coupling increase in $\mathrm{TbPc}_{2}\left(\mathrm{OC}_{11} \mathrm{H}_{21}\right)_{8}$ absorption when there is a coupling between plasmon resonance energy, and the energy needed for transition between the delocalised electrons of the Pc ring to the metal transitions responsible for volatile organic compounds sensing application. These new material properties disclose largely unexplored areas with high scientific and technological potential, thus warranting comprehensive investigations.

\section{Materials and Methods}

\subsection{Preparation of the Magneto-Optical Transducers}

Each magneto-optical transducer consists of a multilayers of $15 \mathrm{~nm} \mathrm{Au} / 6 \mathrm{~nm} \mathrm{Co} / 25 \mathrm{~nm}$ $\mathrm{Au} / 2 \mathrm{~nm}$ Ti deposited on Corning glass substrates (refractive index of 1.51). The $2 \mathrm{~nm}$ thick Ti layer guarantees the adherence between the substrate and the $25 \mathrm{~nm}$ Au layer. All layers were realized by dc magnetron sputtering in an ultrahigh-vacuum chamber with a base pressure of $10^{-9}$ mbar. In these conditions Co layer presents in-plane magnetization $[27,28]$. Co layer buried in specific position in a sandwich of gold layers comes from the need to maximizing the magneto-optical $\Delta \mathrm{R}$ signal as explained in detail in reference [29]. 


\subsection{Preparation of the Magneto-Optical Transducers}

The two compounds used to detect optical difference in the magneto-plasmonic behavior were synthesized according to procedures reported in [30] for the bis-porphyrin derivative and in reference [31] for the terbium phthalocyanine double decker. Both were deposited onto the MP transducers by the Langmuir-Schäfer technique by using a NIMA trough apparatus [32]. A chloroform solution of $\mathrm{TbPc}_{2}\left(\mathrm{OC}_{11} \mathrm{H}_{21}\right)_{8}$, shiwn in Figure 1a, was spread on ultrapure water subphase of a Langmuir trough and the surface pressure (П) vs. area per molecule isotherm curve was recorded during the barrier compression (Figure 2a). The isotherm curve reported in Figure 2a shows three different slope changes suggesting different arrangements of $\mathrm{TbPc}_{2}\left(\mathrm{OC}_{11} \mathrm{H}_{21}\right)_{8}$ molecules spread on the subphase surface. For large values of the surface area, $\mathrm{TbPc}_{2}\left(\mathrm{OC}_{11} \mathrm{H}_{21}\right)_{8}$ molecules do not interact one another. Under the effect of the barriers motion the floating film moves towards the so-called expanded liquid phase [33] and the limiting area calculated in this region (about $110 \AA^{2}$. molecule ${ }^{-1}$ ) suggests that the molecules spread on the subphase surface aggregate [34]. Starting from a surface pressure value of about $12 \mathrm{mN} \cdot \mathrm{m}^{-1}$ a further slope variation is recorded and a limiting area value of $30 \AA^{2}$. Taking in account the high hydrophobic nature of the alkyl substituents, the formation of a uniform floating film must be excluded: the bis-phthalocyanine derivative molecules aggregate in cluster structures. In fact, the calculated value of area per molecule appears too small if compared with the phthalocyanine core theoretical value $\left(290 \AA^{2} \cdot\right.$ molecule $\left.^{-1}\right)$ [35]. Nevertheless, as highlighted by the images recorded by means of a Brewster angle microscope (BAM) (Figure 3), the simultaneous presence of subphase regions covered by $\mathrm{TbPc}_{2}\left(\mathrm{OC}_{11} \mathrm{H}_{21}\right)_{8}$ floating molecules and bare water surface domains are evident at very low values of surface pressure as well as at higher ones confirming a very low homogeneity of the floating film. Brewster angle microscopy allows a real-time observation of the Langmuir floating film without adding any marker molecules that could affect the molecular behavior at the air-subphase interface. The BAM technique utilizes the reflection-free condition that can occur at a specific angle (known as the Brewster angle) for a p-polarized light incident on an air-water interface. According with Snell's law, the Brewster angle depends on the refractive indexes of the materials so changes in presence of a floating film can be observed [36].

Similarly, the Langmuir isotherm and BAM images were also recorded for $\mathrm{CoCoPo}_{2}$ floating film. A chloroform spreading solution containing $0.12 \mathrm{mg} / \mathrm{mL} \mathrm{CoCoPo} 2$ was used (spread volume $150 \mu \mathrm{L}$ ) to form the floating film.
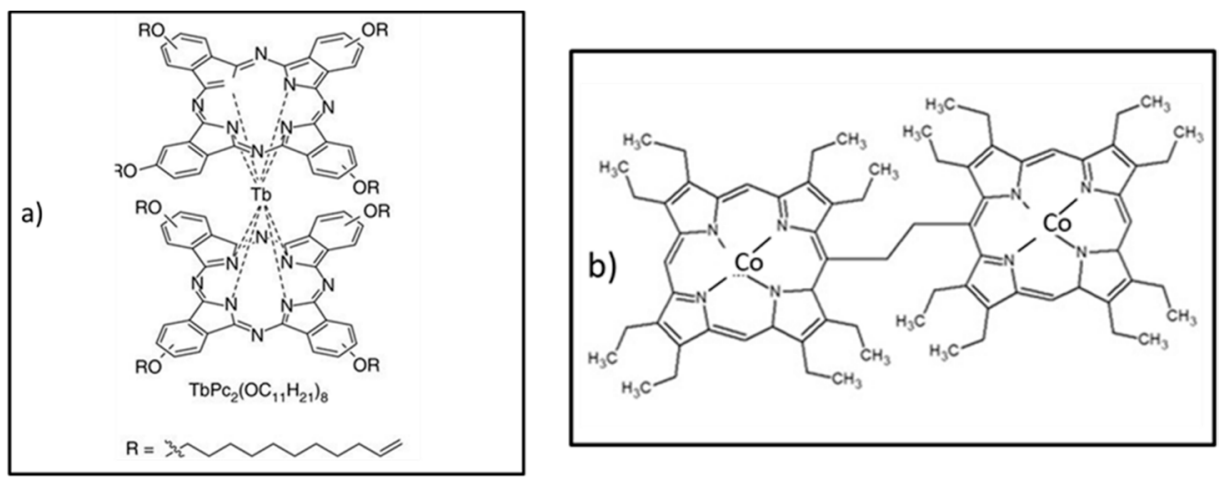

Figure 1. Molecular structures of (a) $\mathrm{TbPc}_{2}\left(\mathrm{OC}_{11} \mathrm{H}_{21}\right)_{8}$ terbium phthalocyanine derivative and (b) bis porphyrin derivative $\mathrm{CoCoPo}_{2}$ used as active molecules. 

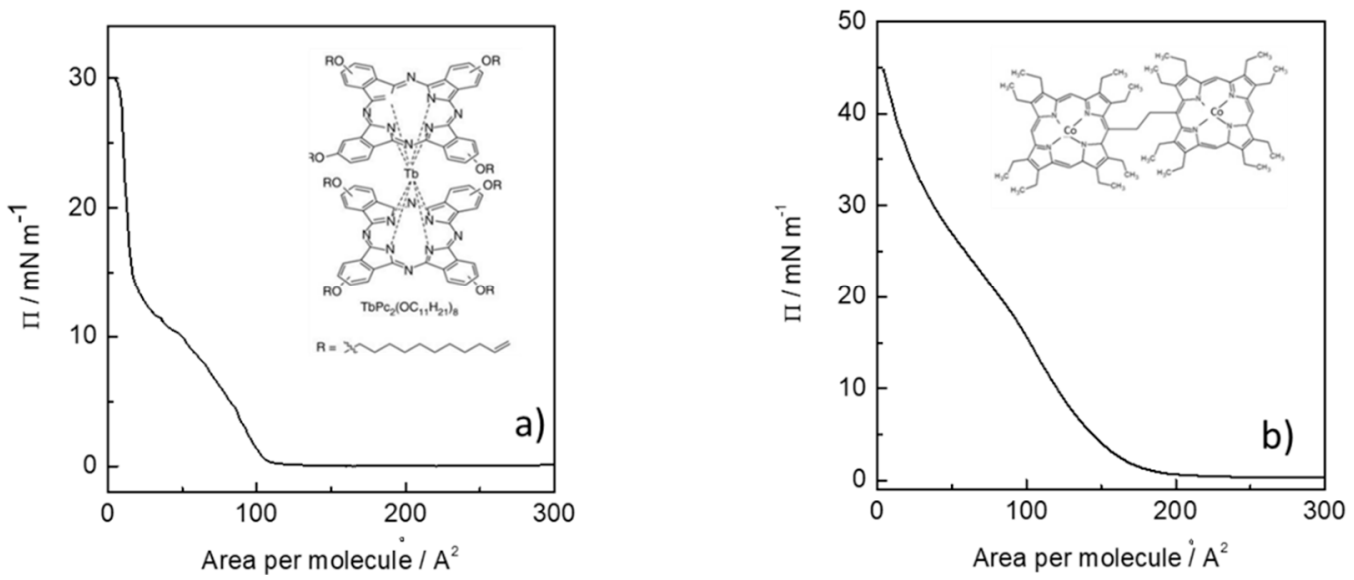

Figure 2. П-area per molecule isotherm curves for the floating film of $\mathrm{TbPc}_{2}\left(\mathrm{OC}_{11} \mathrm{H}_{21}\right)_{8}$ (panel a) and $\mathrm{CoCoPo}_{2}$ (panel b).
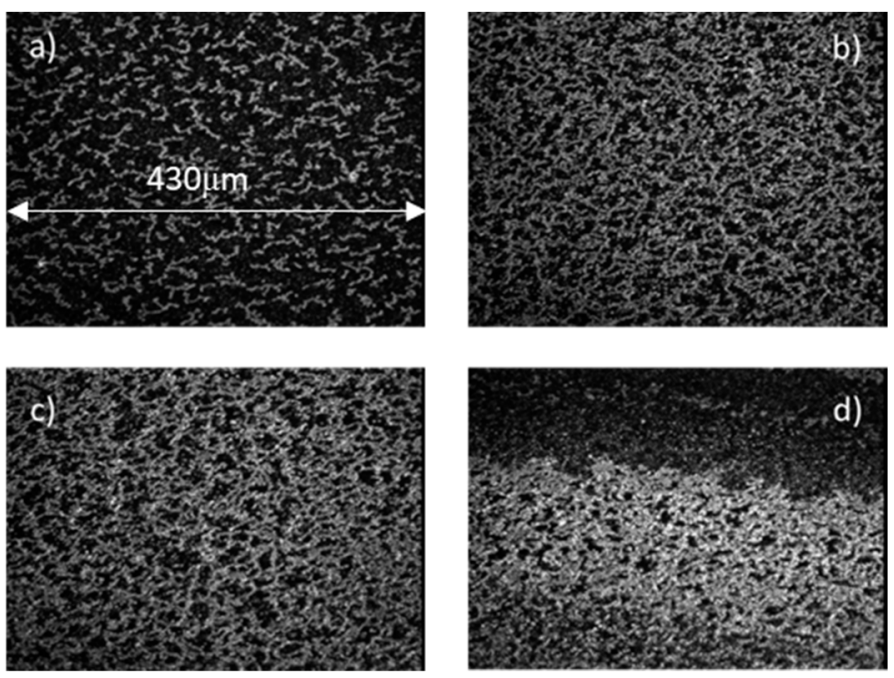

Figure 3. BAM images of $\mathrm{TbPc}_{2}\left(\mathrm{OC}_{11} \mathrm{H}_{21}\right)_{8}$ recorded at different surface pressure values, $4 \mathrm{mN} \cdot \mathrm{m}^{-1}$ (box a), 7.5, 18 and $29 \mathrm{mN} \cdot \mathrm{m}^{-1}$ (boxes $\mathbf{b}-\mathbf{d}$ ). Width of images is $430 \mu \mathrm{m}$.

At least two different slope changes characterize the $\Pi$ vs. $\AA$ graph of the bisporphyrin derivative reported in Figure $2 \mathrm{~b}$. The first one is recorded at the onset at about $200 \AA^{2} \cdot$ molecule $^{-1}$ and the second one at the surface pressure of about $26 \mathrm{mN} \cdot \mathrm{m}^{-1}$. The limiting areas per molecule corresponding to the two isotherm branches are $160 \AA^{2} \cdot \mathrm{molecule}^{-1}$ and $85 \AA^{2} \cdot$ molecule ${ }^{-1}$, respectively. The extrapolated limiting area per molecule of $160 \AA^{2} \cdot$ molecule $^{-1}$ is not compatible with the formation of a floating monolayer $[20,37]$, on the contrary the value $85 \AA^{2} \cdot$ molecule ${ }^{-1}$ fits the area occupied by the $\mathrm{CoCoPo}_{2}$ in edge-on configuration. Nevertheless, BAM images suggest that the subphase surface is not uniformly covered by the floating film. Dendritic structures are formed at low surface pressure value (Figure 4) and they coexist with regions of subphase not covered by the floating film. When the surface pressure increases the observed floating structures become progressively denser (Figure $4 b, c$ ) and at very high surface pressure values different floating film thickness can be observed (Figure $4 \mathrm{~d}$ ). 

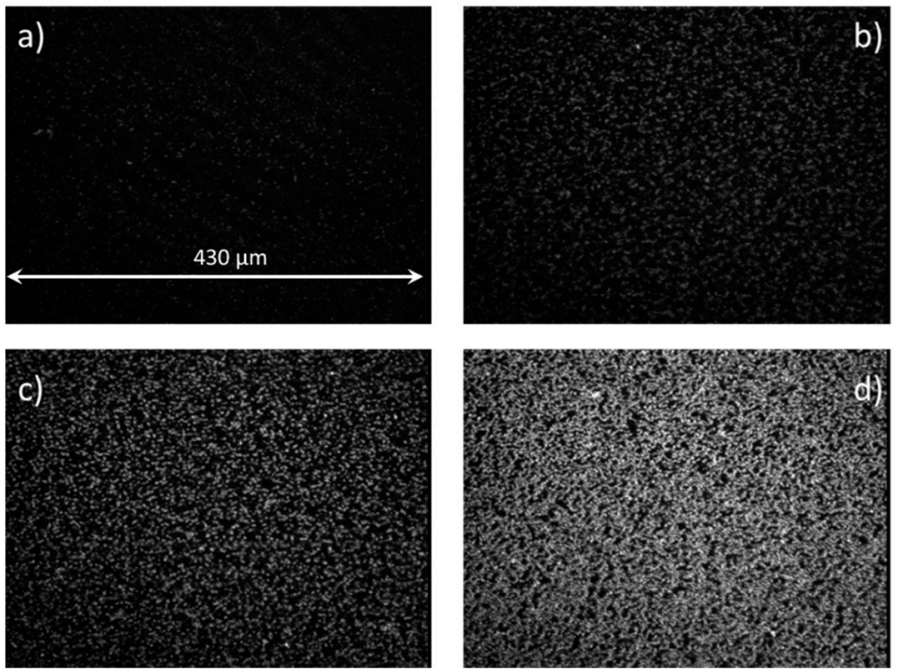

Figure 4. BAM images of $\mathrm{CoCoPo} 2$ recorded at different surface pressure values, $5 \mathrm{mN} \cdot \mathrm{m}^{-1}$ (box a), 12,26 and $34 \mathrm{mN} \cdot \mathrm{m}^{-1}$ (boxes $\mathbf{b}-\mathbf{d}$ ). Width of images is $430 \mu \mathrm{m}$.

\subsection{Morphological and Optical Characterization}

The topography analyses of the films were performed by atomic force microscopy (AFM NT-MDT Spectralight, NT-MDT LLC, Moscow, Russia) and the topographic height signal images were obtained in semi-contact mode. A system equipped with a silicon high resolution conic tip was used (radius of curvature: less than $10 \mathrm{~nm}$; force constant: $17+/-20 \% \mathrm{~N} / \mathrm{m}$ and resonant frequency $230+/-20 \% \mathrm{kHz}$ ). The active LS films were also deposited onto polished quartz slides for UV-visible characterization. Optical absorption measurements, at normal incidence, were conducted with an Agilent Cary 5000 instrument (Santa Clara Campus's main lobby at 5301 Stevens Creek Blvd. in Santa Clara, CA, USA).

\subsection{Plasmonic and Magneto-Plasmonic Characterization in Dry-Air and in Controlled Atmosphere}

Surface plasmons (SP) are electromagnetic waves associated with longitudinal oscillations of the electrons; they propagate along the surface of a conductor, usually a metal. When using the Kretschmann configuration [4] to generate a plasmon, it is sufficient that a p-polarized light wave hits a prism with a high refractive index in total reflection conditions, thus generating an evanescent wave at the base of the prism. This process excites a resonant plasmon if a thin metal film (generally gold) is deposited onto the prism base. In SPR condition, the p-polarized incident light is absorbed by the metal layer and a resonance dip is generated in the reflection spectrum. The resonance condition is described by the following relationship [38]:

$$
\left(2 \pi / \lambda_{\text {inc }}\right) n_{p} \sin \theta=\left(2 \pi / \lambda_{S P P}\right)\left(\varepsilon_{m} n_{s}^{2} /\left(\varepsilon_{m}+n_{s}^{2}\right)^{1 / 2}\right.
$$

where $\lambda_{i n c}$ is the wavelength in free space, $n_{p}$ is the refractive index of the dielectric prism, $\theta$ the incident angle of the illuminating light, $\varepsilon_{m}$ is the permittivity of the metal layer and $n_{s}$ is the refractive index of the dielectric (dry air in our case). $\lambda_{S P P}$ represents the wavelength of the SPP plasmon resonant. By using this equation, we can extract the energy of the SPP resonant plasmon at the metal dielectric interface. In our experimental conditions, standard SPR measurements were performed in a Kretschmann configuration and the wavelength of the excited plasmon resonant was calculated to be $\lambda_{S P P}=675 \mathrm{~nm}$. Au/Co/Au films were deposited onto Corning glass substrates and attached to the base of a BK7 prism $(n=1516)$ with an index matching fluid. A p-polarized He-Ne laser beam $(\lambda=632.8 \mathrm{~nm})$ is directed onto the back surface of the transducers $\mathrm{Au} / \mathrm{Co} / \mathrm{Au}$ thin films through the prism. The intensities of the incident and reflected beams were detected as a function of incidence angle using photodiodes and reflectivity was obtained. The SPR signal manifests itself as a 
dip in the angular dependence of the reflected light at a specific incidence angle above the critical angle. In resonance conditions the light energy is used to excite surface plasmons. It occurs at a characteristic angle of incidence $\left(\theta_{s p p}\right)$, which depends on the thicknesses as well as on the dielectric permittivity of the layers and of the adjacent medium. A shift in the SPR curve give us the opportunity to calculate changes in refractive index in the medium adjacent to the metal film, both real and imaginary part. In our configuration, to monitor the magneto-optical activity in resonant condition, an home- made electromagnet was realized with the magnetic field orientation in transversal configuration (TMOKE). The magnetic field needed to saturate the magnetic domains of the Co layer was fixed at $30 \mathrm{Oe}$ and determined by magneto-optical Kerr effect hysteresis loops in transverse configuration (TMOKE), which is sensitive to in-plane magnetization [27]. The relative variation in the reflected p-polarized light is defined as:

$$
\Delta R=R(+M)-R(-M)
$$

where $R( \pm M)$ are the reflectance of the p-polarized light with the sample magnetically saturated along the positive $(+M)$ and negative $(-M)$ directions of the applied magnetic field. The last equation is translated into a relative variation in the reflected p-polarized light, which is related to the transverse magneto-optic Kerr effect (TMOKE),

$$
\mathrm{TMOKE}=\Delta R / R=[R(+M)-R(-M)] / R(0)
$$

where $R(0)$ represents the p-component of the reflectivity for the demagnetization state. The registered TMOKE signal depends on the light incident angle and on the optical and MO properties of the investigated materials constituting the optical transducer.

\section{Results and Discussion}

\subsection{Morphological Characterization}

Figure 5a,b show the AFM characterization performed onto the two investigated samples containing five monolayers of $\mathrm{TbPc}_{2}\left(\mathrm{OC}_{11} \mathrm{H}_{21}\right)_{8}$ and $\mathrm{CoCoPo}_{2}$ deposited by the LS technique. It is possible to state that, whereas the morphology of the $\mathrm{CoCoPo}_{2}$ shows spherical grains with diameters ranging from a few nanometres to a few dozens, $\mathrm{TbPc}_{2}\left(\mathrm{OC}_{11} \mathrm{H}_{21}\right)_{8}$ highlights the presence of elongated structures that are approximately $500 \mathrm{~nm}$ long and $100 \mathrm{~nm}$ wide with many aggregates. Comparing the BAM observations reported in Figure 4, concerning the formation of the monolayer at the air-water interface, and AFM characterization in Figure 5, even though with a different scale, we can stress that the monolayer keeps the same morphology during the transfer from the air-water interface to the transducer.
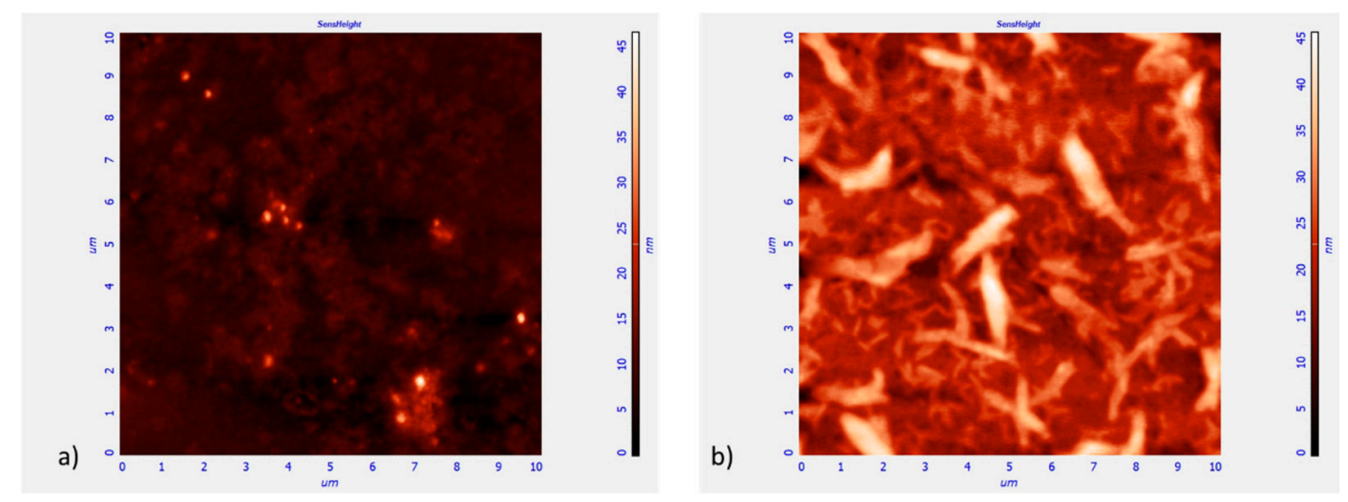

Figure 5. (a) AFM image of the $\mathrm{CoCoPo}_{2}$ and (b) $\mathrm{TbPc}_{2}\left(\mathrm{OC}_{11} \mathrm{H}_{21}\right)_{8} \mathrm{~L}-\mathrm{S}$ layers deposited onto MOSPR transducers.

\subsection{Optical and Magneto-Plasmonic Characterization and Discussion}

In the last years, the coupling interaction between surface plasmon polaritons (SPP) and molecules has been utilized for optical sensing applications. Such interaction is 
generally mediated by electromagnetic (EM) fields, which determine how the optical properties of the molecules are influenced by the presence of the plasmonics system, and vice versa. The molecule is a classical oscillating charge density (usually a point dipole) and the metal is a continuous body characterized by the frequency-dependent dielectric function. In a usual Kretschmann configuration, surface plasmon polaritons and molecular dipoles undergo to an interaction dependent on the plasmon energy and HOMO-LUMO energy level of the organic macrocycle. If the plasmon energy is in resonance with the HOMO-LUMO transition a variation in the angular position and depth of the SPR resonance curve takes place. This behaviour results in a variation in the refractive index real and imaginary part [39], thus demonstrating an energy transfer between plasmon polariton and molecular dipole. Consequently, it is possible to stress that a more reactive chemical environment is generated at the interface macrocycle/dielectric suitable to interact with analytes of interest (VOCs) or gas molecules to detect. Furthermore, in our experimental conditions, the use of magnetic/plasmonic materials as transducers, the presence of a magnetic field in TMOKE configuration, instead of the usual noble metals, allow for an additional degree of freedom for the modulation of electromagnetic field properties and penetration depth of the plasmonic field in the dielectric. In fact, it allows light to interact with the spins of the electrons and to actively manipulate the magnetic properties of such composite material. Moreover, we experimentally demonstrate how these coupling effects artificially enhance sensing properties in organic macrocycles like metallophthalocyanines and metalloporphyrins. The existence of intense local electromagnetic fields around the metal surfaces in contact with the organic molecules, due to excitation of the plasmonic modes magnetically modulated, suggest that they are responsible of the investigated surface-enhanced functionality. Taking into account the penetration depth of the resonant plasmon of about $100 \mathrm{~nm}$, the sensitive region is completely involved in the interaction mechanisms. Consequently, the absorption sites involved in the interaction processes and sensing, due to the presence in one case of $\mathrm{TbPc}_{2}\left(\mathrm{OC}_{11} \mathrm{H}_{21}\right)_{8}$ organic macrocycles, undergo to an energy transfer from plasmon to free $\pi$ electrons of the $\operatorname{TbPc}_{2}\left(\mathrm{OC}_{11} \mathrm{H}_{21}\right)_{8}$ ring and in the same time are undergo to a magnetic modulation. This effect is not active for $\mathrm{CoCoPo}_{2}$; in this circumstance only a variation in the chemical environment takes place with a consequent refractive index real part variation. In the latter case the sensing layer is not electromagnetically reactive to the polar molecules like butylamine. On the contrary, in the case of $\mathrm{TbPc}_{2}\left(\mathrm{OC}_{11} \mathrm{H}_{21}\right)_{8}$, the energy transfer from plasmon to $\pi$ free delocalised electrons of the benzenic ring of the macroclyces gives rise to "hot" absorption sites. In these conditions the polar analytes molecules are more easily captured changing the adsorption desorption processes. In our discussion, we consider first the visible absorption spectrum of $\mathrm{TbPc}_{2}\left(\mathrm{OC}_{11} \mathrm{H}_{21}\right)_{8}$ in chloroform solution reported in Figure 6.

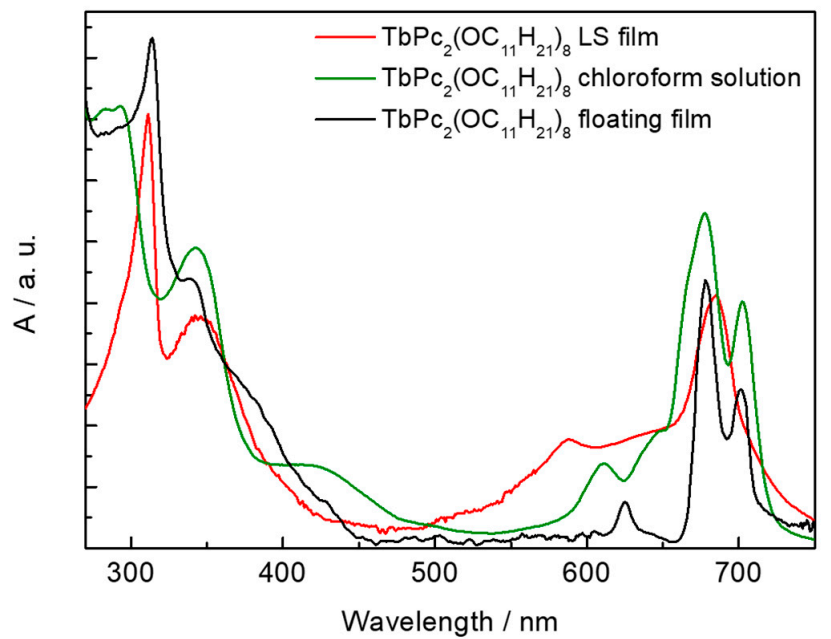

Figure 6. UV-VIS electronic absorption spectra of the $\mathrm{TbPc}_{2}\left(\mathrm{OC}_{11} \mathrm{H}_{21}\right)_{8}$ macrocycle as floating film, in chloroform solution and in L-S thin film form deposited onto silica glass substrate. 
It is characterized by three intense absorption bands located between $300-380 \mathrm{~nm}$ and between $650-750 \mathrm{~nm}$. The reflection spectrum of the floating film at the air-water interface recorded at a surface pressure of $18 \mathrm{mN} \cdot \mathrm{m}^{-1}$ appears very similar to the absorption spectrum of the chloroform solution, just a red shift of the adsorption band at lowest wavelength is observed.

Furthermore, the Langmuir-Schäfer film, obtained transferring five different layers of $\mathrm{TbPc}_{2}\left(\mathrm{OC}_{11} \mathrm{H}_{21}\right)_{8}$ from the Langmuir trough on a quartz substrate at a surface pressure of $18 \mathrm{mN} \cdot \mathrm{m}^{-1}$, shows the same absorption bands of the bis(phthalocyaninato) derivative floating film, testifying that the deposition procedure does not influence the molecular arrangement obtained at the air/subphase interface.

The main absorption peak of the $\mathrm{CoCoPo}_{2}$ chloroform solution reported in Figure 7 can be identified in the Soret band located in the range 350-470 $\mathrm{nm}$. It is interesting to observe that the Soret band is split in two signals at 402 and $433 \mathrm{~nm}$. It is well-known that ethane-bridged bisporphyrin derivatives can assume three different conformational arrangements, the so-called syn-, anti- and tweezer forms. In particular, the signal at $402 \mathrm{~nm}$ can be attributed to the tweezer form of the bis-porphyrin derivative and the $433 \mathrm{~nm}$ is due to the anti-conformer [40].

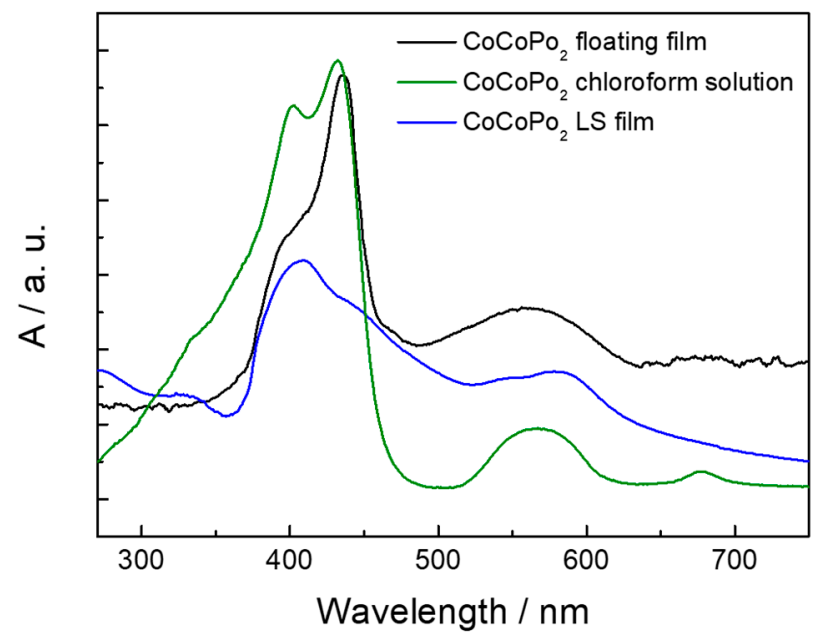

Figure 7. UV-VIS electronic absorption spectra of the $\mathrm{CoCoPo}_{2}$ macrocycle as floating film, in chloroform solution and in L-S thin film form deposited onto silica glass substrate.

Reflection spectrum of the floating film (black line), recorded at $30 \mathrm{mN} \cdot \mathrm{m}^{-1}$, highlights a decrease of the tweezer form while the signal of the anti-conformer becomes predominant [37]. On the contrary, when the floating film is transferred on the quartz substrate by means of the LS method, the tweezer form is induced, and a new band appears as a shoulder at about $375 \mathrm{~nm}$ suggesting that the $\mathrm{CoCoPo}_{2}$ are partially arranged in the closed form (syn-conformer). Anyway, the anti-form does not disappear as confirmed by the signal at $440 \mathrm{~nm}$.

Figure 8 reports the optical absorption curves of the two analysed macrocycles deposited in thin film form and related to a single monolayer of both systems. It is possible to observe a specific absorption peak of the $\mathrm{TbPc}_{2}\left(\mathrm{OC}_{11} \mathrm{H}_{21}\right)_{8}$ centred at about $670 \mathrm{~nm}$. This absorption peak is in resonance with the plasmon energy wave propagating at the gold/dielectric interface due to the evanescent wave generated from the Kretschmann configuration by a HeNe $(633 \mathrm{~nm})$ incident laser beam. In the same spectral region, the $\mathrm{CoCoPo}_{2}$ does not evidence any optical absorption and consequently any resonance or coupling can be generated between the propagating plasmon and the macrocycle molecules. 


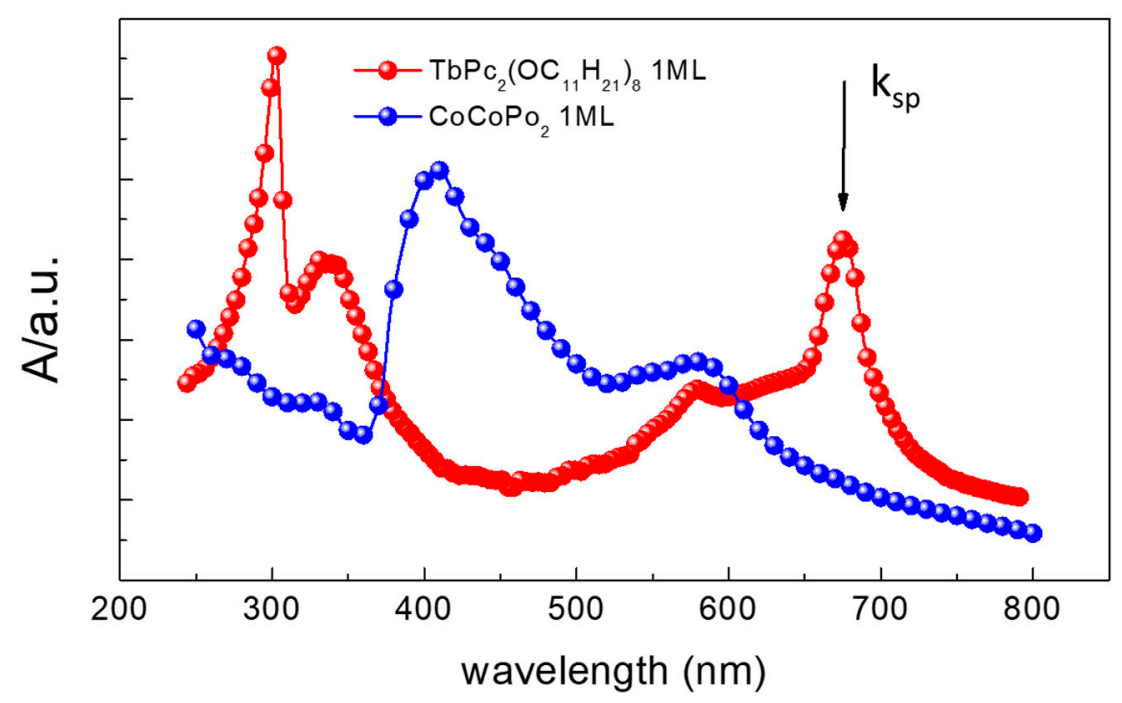

Figure 8. UV-VIS electronic absorption spectra comparison of $\mathrm{TbPc}_{2}\left(\mathrm{OC}_{11} \mathrm{H}_{21}\right)_{8}$ and $\mathrm{CoCoPo}_{2}$ monolayer deposited onto silica glass substrate. The position of the transducers surface plasmon resonance energy coupling with electronic transition of the $\mathrm{TbPc}_{2}\left(\mathrm{OC}_{11} \mathrm{H}_{21}\right)_{8}$ is evidenced.

Figure 8 evidences this different optical behavior regarding the position in terms of energy of the plasmon probe wavevector $\mathrm{k}_{\mathrm{sp}}$. From the plasmonic curves reported in Figure 9a, relative to the five $\mathrm{CoCoPO}_{2}$ monolayers, we stress a more limited energy transfer between plasmon and $\mathrm{CoCoPo}_{2}$ molecule due to the absence of the overlap between plasmon dipole energy and electronic molecular transition energies.
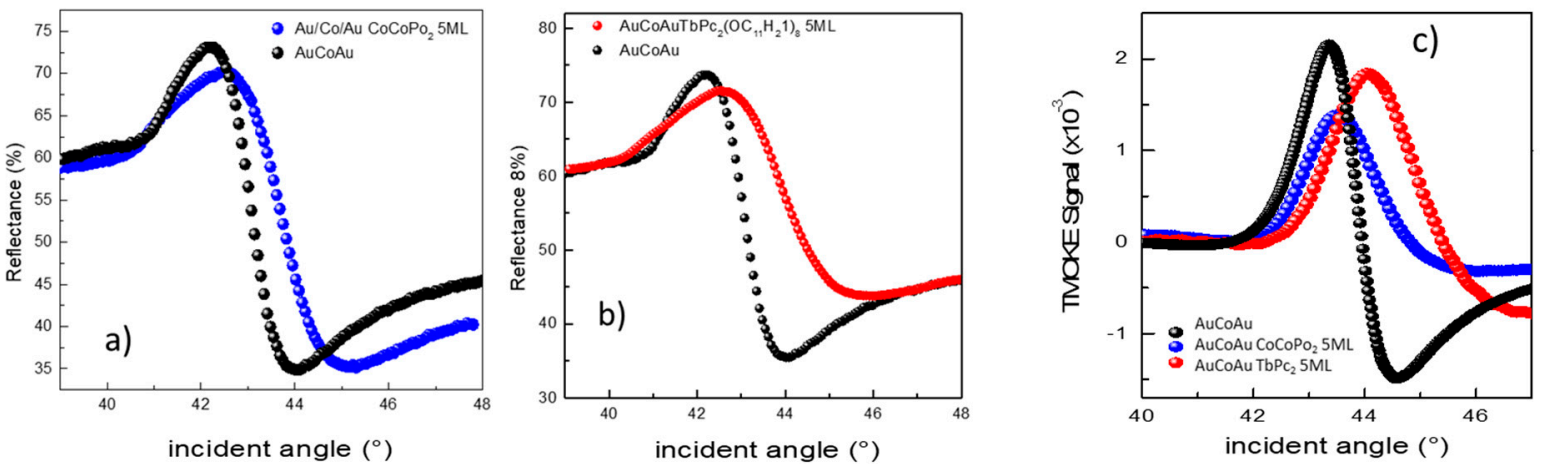

Figure 9. (a,b) Experimental reflectivity curves (SPR) vs. angle of incidence of $\mathrm{TbP}_{2}\left(\mathrm{OC}_{11} \mathrm{H}_{21}\right)_{8}$ and $\mathrm{CoCoPo}$ macrocycles. (c) Magneto-plasmonic curves (TMOKE signal) of $\mathrm{Au} / \mathrm{Co} / \mathrm{Au}$ transducers covered by 5 monolayers (5ML) of $\mathrm{TbPc}_{2}\left(\mathrm{OC}_{11} \mathrm{H}_{21}\right)_{8}$ and $\mathrm{CoCoPo}_{2}$, respectivley.

There is in fact a red-shift in the SPR curve corresponding to a variation of the dielectric constant real part and only a slight variation in the depth of the peak, linked to the imaginary part of the dielectric constant and therefore to energy absorption phenomena. On the contrary, from the plasmonic curves reported in Figure $9 \mathrm{~b}$ and relative to five $\mathrm{TbPc}_{2}\left(\mathrm{OC}_{11} \mathrm{H}_{21}\right)_{8}$ monolayers, we stress variation due to the refractive index of the macrocycles adlayer but also a a significant energy transfer between propagating plasmon and $\mathrm{TbPc}_{2}\left(\mathrm{OC}_{11} \mathrm{H}_{21}\right)_{8}$ molecule.

In fact, a red-shift of the SPR signal is evidenced, but also a substantial decrease in the depth of the SPR peak. A dipole-dipole interaction between plasmonic dipole energy and free $\pi$ electrons of the phthalocyanine ring takes place. The physical effects of this interaction are evidenced also in the MOSPR curves reported in Figure 9c.

In both cases the magneto-optical TMOKE signal probes are characterized by a sharp resonance like angular behaviour at around $45^{\circ}$, i.e., when the SPP is excited, but the 
experimental effects are significantly different in terms of simple resonant schift and dept of the resonant curve. The resonant angle $\theta_{\text {MOSPR }}$ corresponding to the activation of MOSPR signal is more precisely visualized by plotting the quantity 1 / I TMOKE I. Plotting the data in this manner makes much easier to visualize the gain in precision and response. In Figure 10a the absolute value of $1 /$ ITMOKE I as a function of the incidence angle $\theta$ for $\mathrm{TbPc}_{2}\left(\mathrm{OC}_{11} \mathrm{H}_{21}\right)_{8}$ and $\mathrm{CoCoPo}_{2}$ molecules is reported. The spectra of $1 /|\mathrm{MOSPR}|$ reported in Figure 10 evidences a very narrow signal FWHM giving the opportunity to discriminate between the nature of the single monolayer deposited onto the surface of transducer, pointing out at this point the ability of the system to discriminate between molecules with $\pi-\pi^{*}$ electronic transitions close to the resonant plasmon or, on the contrary as for $\mathrm{CoCoPo}_{2}$, far away. It also demonstrates the ability of the system to sense unequivocal the presence of five monolayer through a laser spot probe (1 $\mathrm{mm}$ in size) and through an high magneto-plasmonic signal, so that we can stress to have taken the road towards the realization of a system with improved molecular sensing also under five monolayers, thus able to monitor even the single monolayer (single molecule layer) deposited onto the MOSPR transducer. Furthermore, we can also demonstrate that in terms of VOCs sensing capability comparison between the two configuration, Figure $10 \mathrm{~b}$ puts in evidence the sensing responses in term of $1 /$ I TMOKE I signal when the sensing molecules are in contact with a typical VOC like butylamine. The rationale is that the interactions between VOCs and organic macrocycles are favoured in the case of coupling between resonant plasmons energy and the $\pi$ free electron of the phthalocyanine ring in comparison with to the decoupling situation relative to the porphyrins, consequently the sensing signal increases.

Despite the interesting results obtained, we need also to take into account the extinction coefficients and the direction of the dipole (related to the orientation of the molecules in the monolayer) which may also play a significant role in the sensing mechanism. Moreover the frontier orbital energies have to be estimated by using other methods taking into account that the two analysed compounds have different molecular structures and more further studies will be done by our group to support the claims.

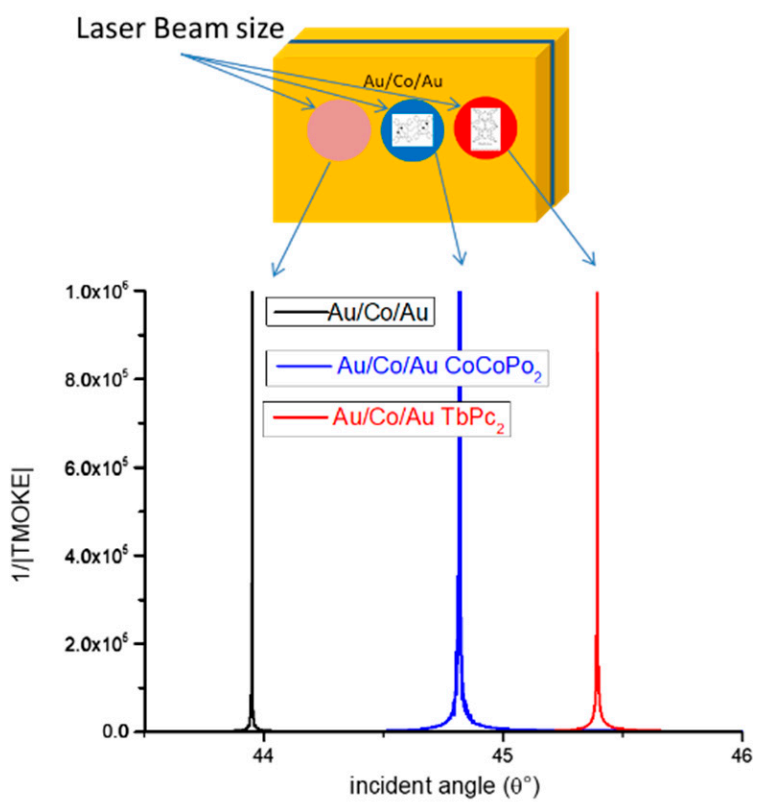

(a)

Figure 10. Cont. 


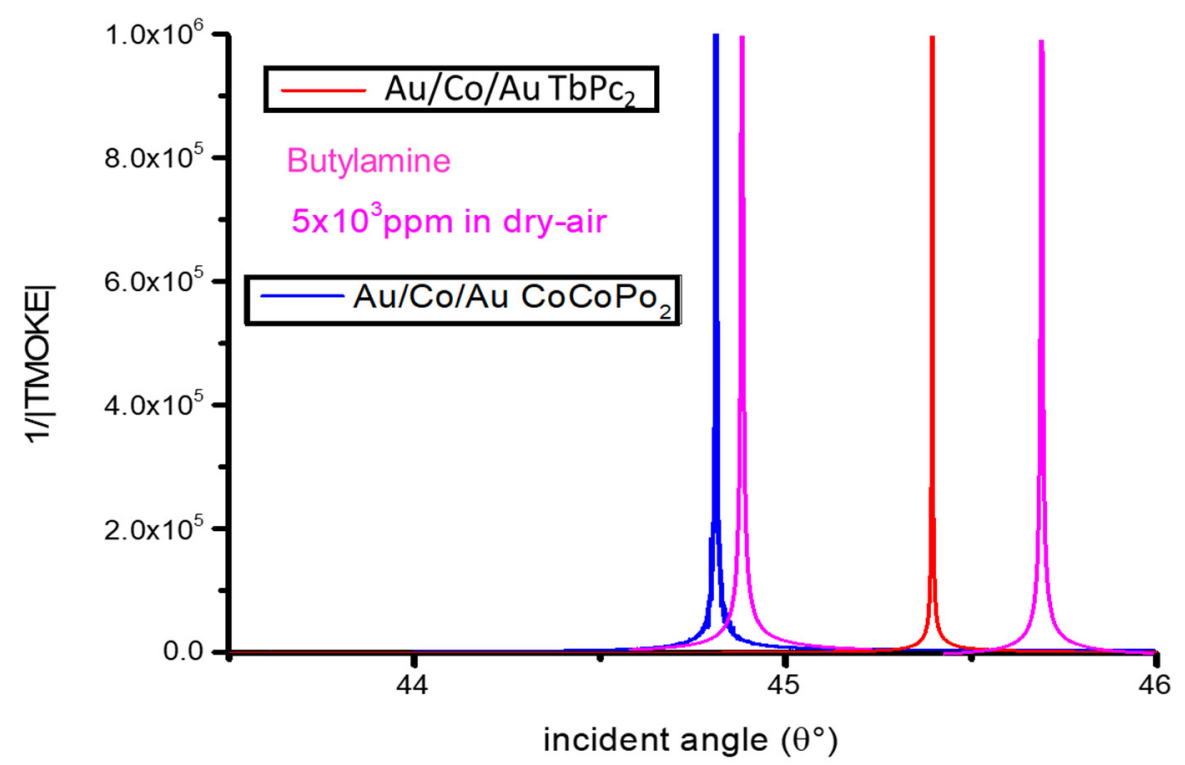

(b)

Figure 10. (a) $1 /$ I TMOKE I signal vs. incidence angle of the $\mathrm{Au} / \mathrm{Co} / \mathrm{Au}$ transducer alone compared with the same signals acquired in the presence of a single monolayer of $\mathrm{CoCoPo}_{2}$ and $\mathrm{TbPc}_{2}\left(\mathrm{OC}_{11} \mathrm{H}_{21}\right)_{8}$ macrocycles. The coloured circles represent the investigating laser size $(<0.5 \mathrm{~mm})$. (b) 1 / I TMOKE I signals vs. incidence angle in Kretschmann configuration for $\mathrm{CoCoPo}_{2}$ and $\mathrm{TbPc}_{2}\left(\mathrm{OC}_{11} \mathrm{H}_{21}\right)_{8}$ monolayers interacting with butylamine vapours, respectively.

\section{Conclusions}

Optical and magneto-optical surface plasmon resonance (MOSPR) characterizations have been performed on layers of two organic macrocycles- $\mathrm{TbPc}_{2}\left(\mathrm{OC}_{11} \mathrm{H}_{21}\right)_{8}$ phthalocyanine and $\mathrm{CoCoPo}_{2}$ porphyrin - both deposited by the Langmuir-Schäfer (LS) technique. Investigations of the MOSPR properties, in Kretschmann configuration by angular modulation, demonstrate an interesting capacity to discriminate the two organic macrocycles with absorption electronic transitions resonant with surface plasmon energy in Kretschmann configuration. A sensitivity increase was detected by MOSPR sensing probe if an overlap between the plasmonic probe energy and the electronic transitions of the PC ring free electrons of the investigated macrocycles are in resonance condition. In this experimental condition we emphasize how, most likely, single molecule detection can be obtained by using 1 / I MOSPR | magneto-plasmonic signal. Moreover, an increase in the VOCs sensing capacity is demonstrated when the propagating plasmon energy is in resonance with the HOMO-LUMO transitions. Further analysis by using different metal-coordinated organic macrocycles tuned to the plasmon resonance energy are in progress in order to confirm this effect. The related results will be the object of a future paper, together to the detection limit concentration of $n$-butylamine using the complex system and reversibility of the TMOKE signal in the presence or absence of butylamine vapors.

Author Contributions: This manuscript was written through contributions of all authors. A.C. performed the preparation of the transducers; S.B., G.G. and L.V. were involved in the LB deposition and optical characterization. V.B. synthetized and characterized organic macrocycles. Atomic force microscopy measurements and morphology discussion were optimised by D.L. Magneto-optical measurements and gas sensing tests were performed by M.G.M. and R.R. All the authors contributed to the discussion of data, designed, and discussed the whole experiments with a strong contribution in revision of the paper. All authors have read and agreed to the published version of the manuscript.

Funding: V.B. acknowledges the financial support from the European Union's H2020-FETOPEN grant 828779 (INITIO). 
Acknowledgments: The authors thank Jianzhuang Jiang (Beijing Key Laboratory for Science and Application of Functional Molecular and Crystalline Materials, Department of Chemistry, University of Science and Technology, Beijing, China) who approved the kind and friendly supply of Tb bisphthalocyanine used in this manuscript and G. Montagna and E. Melissano for technical support during the experiments.

Conflicts of Interest: The authors declare no conflict of interest.

\section{References}

1. Sonntag, M.D.; Klingsporn, J.M.; Zrimsek, A.B.; Sharma, B.; Ruvuna, L.K.; Van Duyne, R.P. Molecular plasmonics for nanoscale spectroscopy. Chem. Soc. Rev. 2014, 43, 1230. [CrossRef] [PubMed]

2. Zheng, Y.B.; Kiraly, B.; Weiss, P.S.; Huang, T.J. Molecular plasmonics for biology and nanomedicine. Nanomedicine 2012, 7, 5. [CrossRef] [PubMed]

3. Csaki, A.; Schneider, T.; Wirth, J.; Jahr, N.; Steinbrück, A.; Stranik, O.; Garwe, F.; Müller, R.; Fritzsche, W. Molecular plasmonics: Light meets molecules at the nanoscale. Phil. Trans. R. Soc. A 2011, 369, 3483-3496. [CrossRef] [PubMed]

4. Manera, M.G.; Ferreiro-Vila, E.; García-Martín, J.M.; Cebollada, A.; García-Martín, A.; Giancane, G.; Valli, L.; Rella, R. Enhanced magneto-optical SPR platform for amine sensing based on Zn porphyrin dimers. Sens. Actuators B Chem. 2013, 182, 232-238. [CrossRef]

5. Kaur, G.; Lewis, J.S.; van Oijen, A.M. Shining a spotlight on DNA: Single-molecule methods to visualize DNA. Molecules 2019, 24, 491. [CrossRef] [PubMed]

6. Rissin, D.M.; Kan, C.W.; Campbell, T.G.; Howes, S.C.; Fournier, D.R.; Song, L.; Piech, T.; Patel, P.P.; Chang, L.; Rivnak, A.J.; et al. Single-molecule enzyme-linked immunosorbent assay detects serum proteins at subfemtomolar concentrations. Nat. Biotechnol. 2010, 28, 595-599. [CrossRef] [PubMed]

7. Pavlov, A.M.; Gabriel, S.A.; Sukhorukov, G.B.; Gould, D.J. Improved and targeted delivery of bioactive molecules to cells with magnetic layer-by-layer assembled microcapsules. Nanoscale 2015, 7, 9686-9693. [CrossRef]

8. Baselt, D.R.; Lee, G.U.; Natesan, M.; Metzger, S.W.; Sheehan, P.E.; Colton, R.J. A biosensor based on magnetoresistance technology. Biosens. Bioelectron. 1998, 13, 731-739. [CrossRef]

9. Buznikov, N.A.; Safronov, A.P.; Orue, I.; Golubeva, E.V.; Lepalovskij, V.N.; Svalov, A.V.; Chlenova, A.; Kurlyandskaya, G.V. Modelling of magnetoimpedance response of thin film sensitive element in the presence of ferrogel: Next step toward development of biosensor for in-tissue embedded magnetic nanoparticles detection. Biosens. Bioelectron. 2018, 117, 366-372. [CrossRef]

10. Rodriguez-Mendez, M.; de Saja, J.A. Chapter 3, Molecular materials for gas sensors and sensor arrays. In Advanced Nanomaterials for Inexpensive Gas Microsensors; Synthesis, Integration and Applications; Elsevier: Amsterdam, The Netherlands, 2020; pp. 37-54. [CrossRef]

11. Dong, Z.; Kong, X.; Wu, Y.; Zhang, J.; Chen, Y. High-sensitive room-temperature $\mathrm{NO}_{2}$ sensor based on a soluble ntypephthalocyanine semiconductor. Inorg. Chem. Commun. 2017, 77, 18-22. [CrossRef]

12. Çapan, R. Porphyrin langmuir-blodgett thin film for organic vapor detection. J. Phys. Sci. Appl. 2019, 9, $15-24$.

13. Castillero, P.; Roales, J.; Lopes-Costa, T.; Sánchez-Valencia, J.R.; Barranco, A.; González-Elipe, A.R.; Pedrosa, J.M. Optical gas sensing of ammonia and amines basedon protonated porphyrin $/ \mathrm{TiO}_{2}$ Composite thin films. Sensors 2017, 17, 24. [CrossRef] [PubMed]

14. Krichevsky, D.; Zasedatelev, A.; Tolbin, A.Y.; Luchkin, S.; Karpo, A.B.; Krasovskii, V.I.; Tomilova, L.G. Highly transparent low-symmetry zinc phthalocyanine-based monolayersfor $\mathrm{NO}_{2}$ gas detection. Thin Solid Films 2017, 642, 295-302296. [CrossRef]

15. Ali, A.M.; Said, D.A.; Khayyat, M.; Boustimi, M.; Seoudi, R. Improving the efficiency of the organic solar cell (CuPc/C60) via PEDOT: PSS as a photoconductor layer doped by silver nanoparticles. Results Phys. 2020, 16, 102819. [CrossRef]

16. Gao, H.H.; Sun, Y.; Li, S.; Ke, X.; Cai, Y.; Wan, X.; Zhang, H.; Li, C.; Chen, Y. An all small molecule organic solar cell based on a porphyrin donor and a non-fullerene acceptor with complementary and broad absorption. Dye. Pigment. 2020, 176, 108250. [CrossRef]

17. Matsuo, Y.; Ogumi, K.; Jeon, I.; Wang, H.; Nakagawa, T. Progress in porphyrin- and phthalocyanine-containing perovskite solar cells. RSC Adv. 2020, 10, 32678-32689. [CrossRef]

18. Avossa, J.; Paolesse, R.; Di Natale, C.; Zampetti, E.; Bertoni, G.; De Cesare, F.; Scarascia-Mugnozza, G.; Macagnano, A. Electrospinning of polystyrene/polyhydroxybutyrate nanofibers doped with porphyrin and graphene for chemiresistor gas sensors. Nanomaterials 2019, 9, 280. [CrossRef]

19. Duan, X.; Zhang, Y.; Wang, H.; Dai, F.; Yang, G.; Chen, Y. A phthalocyanine sensor array based on sensitivity and current changes for highly sensitive identification of three toxic gases at ppb levels. New J. Chem. 2020, 44, 13240-13248. [CrossRef]

20. Klyamer, D.; Bonegardt, D.; Basova, T. Fluoro-substituted metal phthalocyanines for active layers of chemical sensors. Chemosensors 2021, 9, 133. [CrossRef]

21. Acikbas, Y.; Erdogan, M.; Capan, R.; Ozkaya, C.; Baygu, Y.; Kabay, N.; Gok, Y. Preparation of Zinc (II) phthalocyanine-based LB thin film: Experimental characterization, the determination of some optical properties and the investigation of the optical sensing ability. Optik 2021, 245, 167661. [CrossRef]

22. Kharitonova, N.V.; Maiorova, L.A.; Koifman, O.I. Aggregation behavior of unsubstituted magnesium porphyrazine in monolayers at air-water interface and in Langmuir-Schaefer films. J. Porphyr. Phthalocyanines 2018, 22, 509-520. [CrossRef] 
23. Kazak, A.; Marchenkova, M.; Smirnova, A.; Dubinina, T.; Seregin, A.; Rogachev, A.; Usoltseva, N. Thin-film materials based on phthalocyanine derivatives: Structure and physico-chemical properties. ITM Web Conf. 2019, 30, 08006. [CrossRef]

24. Abdulla, S.; Pullithadathil, B. Unidirectional langmuir-blodgett-mediated alignment of polyaniline-functionalized multiwalled carbon nanotubes for $\mathrm{NH}_{3}$ gas sensor applications. Langmuir 2020, 36, 11618-11628. [CrossRef] [PubMed]

25. Manera, M.G.; Rella, R. Improved gas sensing performances in SPR sensors by transducers activation. Sens. Actuators B Chem. 2013, 179, 175-186. [CrossRef]

26. Wang, L.; Guo, S.; Zhou, K.; Ma, W. Control of the molecular orientation in small molecule-based organic photovoltaics. Sustain. Energy Fuels 2020, 4, 4934-4955. [CrossRef]

27. Uzurano, G.; Yabuuchi, Y.; Ishiura, R.; Yoneya, M.; Nagano, S.; Kajii, H.; Fujii, A.; Ozaki, M. Molecular orientation, and electrical properties in tert-butylated phthalocyanine thin film fabricated by uniaxial solution coating. Electron. Comm. JPN 2021, 104, 113-119. [CrossRef]

28. Rizal, C. Magneto-optic surface plasmon resonance Ti/Au/Co/Au/Pc configuration and sensitivity. Magnetochemistry 2018, 4, 35. [CrossRef]

29. González-Díaz, J.; García-Martín, A.; Armelles, G.; García-Martín, J.; Clavero, C.; Cebollada, A.; Lukaszew, R.; Skuza, J.; Kumah, D.; Clarke, R. Surface-magnetoplasmon nonreciprocity effects in noble-metal/ferromagnetic heterostructures. Phys. Rev. B 2007, 76, 153402. [CrossRef]

30. Borovkov, V.V.; Lintuluoto, J.M.; Inoue, Y. Synthesis of Zn-, Mn-, and Fe-containing mono- and heterometallated ethanediylbridged porphyrin dimers. Helv. Chim. Acta 1999, 82, 919-934. [CrossRef]

31. Jiang, J.; Liu, R.C.W.; Mak, T.C.W.; Chan, T.W.D.; Ng, D.K.P. Synthesis, spectroscopic and electrochemical properties of substituted bis(phthalocyaninato)lanthanide(III) complexes. Polyhedron 1997, 16, 515-520. [CrossRef]

32. Bodik, M.; Maxian, O.; Hagara, J.; Nadazdy, P.; Jergel, M.; Majkova, E.; Siffalovic, P. Langmuir-Scheaffer technique as a method for controlled alignment of 1D materials. Langmuir 2020, 36, 4540-4547. [CrossRef]

33. Hussain, S.A.; Dey, B.; Bhattacharjee, D.; Mehtab, N. Unique supramolecular assembly through Langmuir-Blodgett (LB) technique. Helyon 2018, 4, e01038. [CrossRef] [PubMed]

34. Komeda, T.; Katoh, K.; Yamashita, M. Double-decker phthalocyanine complex: Scanning tunneling microscopy study of film formation and spin properties. Prog. Surf. Sci. 2014, 89, 127-160. [CrossRef]

35. Valkova, L.A.; Zyablov, S.V.; Erokhin, V.V.; Koifman, O.I. Nanoaggregates in floating layers of azaporphyrins. J. Porphyr. Phthalocyanines 2010, 14, 513-522. [CrossRef]

36. Vittorino, E.; Giancane, G.; Bettini, S.; Valli, L.; Sortino, S. Bichromophoric multilayer films for the light-controlled generation of nitric oxide and singlet oxygen. J. Mater. Chem. 2009, 19, 8253-8258. [CrossRef]

37. Giancane, G.; Borovkov, V.; Inoue, Y.; Conoci, S.; Valli, L. Syn-anti conformation switching of a bis-porphyrin derivative at the air-water interface and in the solid state as an effective tool for chemical sensing. Soft Matter 2013, 9, 2302-2307. [CrossRef]

38. Homola, J.; Yee, S.S.; Gauglitz, G. Surface plasmon resonance sensors: Review. Sens. Actuators B Chem. 1999, 54, 3-15. [CrossRef]

39. Hong, X.; Lapsley, M.C.; Shang, Y.; Hall, E.A.H. Mapping minimum reflection distribution of surface plasmon resonance with a complex refractive index. Anal. Methods 2016, 8, 8299.

40. Buccolieri, A.; Hasan, M.; Bettini, S.; Bonfrate, V.; Salvatore, L.; Santino, A.; Borovkov, V.; Giancane, G. Ethane-bridged bisporphyrin conformational changes as an effective analytical tool for nonenzymatic detection of urea in the physiological range. Anal. Chem. 2018, 90, 6952-6958. [CrossRef] 